Check for updates

Cite this: RSC Adv., 2018, 8, 29078

\title{
Lactobionic acid-functionalized polyethersulfone hollow fiber membranes promote HepG2 attachment and function
}

\begin{abstract}
Surendra Kumar Verma, ${ }^{a}$ Akshay Modi, (DD a Ashwin Dravid ${ }^{a}$ and Jayesh Bellare (D) *abc
Surface modification of polyethersulfone hollow fibers, which are important in bio-artificial liver, is increasingly used to improve biocompatibility and promote the adhesion and proliferation of hepatocytes resulting in improved cell functionality. Hepatocytes are anchorage-dependent cells, and membrane surface modification enhances the hepatic cell adhesion and proliferation. Specific interaction of the asialoglycoprotein receptor on hepatocyte cell surfaces with a galactose moiety enhances the attachment of the cells on a biocompatible substrate. In this study, the outer surface of the polyethersulfone (P) hollow fiber membranes (HFMs) was chemically modified by covalent coupling with lactobionic acid (LBA). The energy dispersive $X$-ray spectrometry elemental mapping, attenuated total reflectance-Fourier transform infrared spectroscopy, and $X$-ray photoelectron spectroscopy confirmed the LBA-coupling on the outer surface of P-LBA HFMs. Hemocompatibility study indicated the suitability of the modified membranes with human blood. These membranes showed remarkably improved biocompatibility with human primary mesenchymal stem cells and HepG2 cells. Characteristic multi-cellular spheroids of HepG2 cells were observed under scanning electron and confocal microscopy. HepG2 cell functional activity was measured by quantifying the urea synthesis, albumin secretion and glucose consumption in the culture media, which indicated the improved HepG2 functions. These experimental results clearly suggest the potentiality of these LBA-modified P HFMs as a suitable biocompatible substrate for promoting HepG2 attachment and function leading to their application in bioreactors and bio-artificial liver devices.
\end{abstract}

\author{
Received 15th March 2018 \\ Accepted 7th August 2018 \\ DOI: $10.1039 / \mathrm{c} 8 \mathrm{ra} 02282 \mathrm{~h}$ \\ rsc.li/rsc-advances
}

\section{Introduction}

Both natural and synthetic biomaterials of different compositions are being explored for tissue engineering applications. Synthetic polymers are chosen because they can be easily chemically modified and mass-produced as per the desired application. ${ }^{1}$ Polyethersulfone (PES/P) is a nondegradable, biocompatible polymer membrane material, which is extensively being used in hemodialysis, ultrafiltration, gas separation and bioreactor technology. ${ }^{2-4}$ PES has excellent thermal stability and chemical resistance. ${ }^{5}$

Surface properties of different materials including implanted devices, scaffolds, cell culture supports, and bioreactors control cell and host responses. ${ }^{6}$ Over the last few years, most of the advancement in the field of biomaterials has been focused

\footnotetext{
${ }^{a}$ Department of Chemical Engineering, Indian Institute of Technology Bombay, Mumbai, 400076, India. E-mail: jb@iitb.ac.in; Fax: +91-22-2572-6895; Tel: +91-222576-7207

${ }^{b}$ Centre for Research in Nanotechnology \& Sciences, Indian Institute of Technology Bombay, Mumbai, 400076, India

${ }^{c}$ Wadhwani Research Center for Bioengineering, Indian Institute of Technology Bombay, Mumbai, 400076, India
}

on activating these different materials biologically, which would regulate cell behavior due to the interactions with specific cell membrane receptors via incorporation of ligand molecules. ${ }^{7}$ Membrane surface modification, involving both chemical modification and physical coating, enhances the hepatocytic cell adhesion and proliferation. Hepatocytes are known as anchorage-dependent cells, and their viability and differentiated function maintenance are highly sensitive to the extracellular matrix (ECM) environment. ${ }^{8}$ Very recently, the chitosanmodified composite polysulfone hollow fiber membranes (HFMs) showed enhanced attachment and proliferation of HepG2 cells exhibiting characteristic three dimensional multicellular spheroid morphology. ${ }^{9}$ In another study, PES HFMs were surface functionalized with glutaraldehyde-crosslinked gelatin, which supported the HepG2 cells attachment, growth, and proliferation. ${ }^{\mathbf{1 0}}$ Galactose-derived Pluronic F68 (F68-gal) was adsorbed on polyvinylidene fluoride (PVDF) membrane promoted hepatocyte attachment and proliferation, which resulted in high albumin synthesis and P450 1A1 activity as compared to unmodified and collagen-coated PVDF membranes. $^{\mathbf{1 1}}$

Cell surface receptors of hepatocyte recognize and bind molecules with exposed galactose, $N$-acetylgalactosamine, or 
glucose residues. ${ }^{\mathbf{1 2}}$ It is reported in the literature, that galactosylated alginate capsules binds with hepatocytes via the patch of asialoglycoprotein receptor (ASGPR). The galactosemodified substrate enhanced liver functions of hepatocytes compared with those in alginate capsules. ${ }^{13}$ Interaction between cells and extracellular matrix (ECM) permits cell attachment and provides regulatory signals to cells through adhesion. ${ }^{\mathbf{1 4}}$ There are a variety of cell types that have been reported to adhere to PES, including endothelial cells, fibroblasts, osteoblasts, epithelial cells and keratinocytes.,10 HFM based substrates are preferred because they provide threedimensional microenvironment, immunoisolation, continuous flow, large capacity and scale up benefit over other systems. $^{15}$

In the present study, lactobionic acid (LBA) as ASGPR ligand was covalently grafted on the outer surface of P HFMs to promote HepG2 cells adhesion and proliferation. LBA was covalently attached on the outer surface of HFMs by a facile method via amination of P HFMs. The modified HFMs are denoted as P-LBA HFMs, which were characterized by environmental scanning electron microscopy (ESEM), energy-dispersive $\mathrm{X}$-ray spectroscopy (EDS) elemental mapping, attenuated total reflectance-Fourier transform infra-red (ATR-FTIR) spectroscopy, and X-ray photoelectron spectroscopy (XPS). The hemocompatibility of HFM samples was also evaluated using human blood. The developed membranes were evaluated for biocompatibility and cytocompatibility using human primary mesenchymal stem cells and HepG2 cells. HepG2 specific cell functional studies were performed to evaluate the cell activity.

\section{Materials and methods}

\subsection{Materials}

PES (Ultrason E $6020 \mathrm{P}$ ), and $N$-methyl-2-pyrrolidone (NMP) were procured from BASF (Germany), and Spectrochem (India), respectively, and used without any further purification. LBA was procured from Sigma-Aldrich (United States). $\mathrm{N}$ Hydroxysuccinimide (NHS), 1-(3-dimethylaminopropyl)-3ethylcarbodiimide hydrochloride (EDC) and chloromethyl methyl ether (CMME) were bought from Spectrochem Pvt. Ltd. (India). 2-Morpholine ethane sulphonic acid (MES) was purchased from Sisco Research Laboratories (India). Tin chloride $\left(\mathrm{SnCl}_{4}\right)$ was procured from Sigma-Aldrich (United States). Hexane and ethylene diamine (EDA) were purchased from Merck Chemicals (India) and phosphate buffered saline (PBS) was bought from HiMedia Laboratories Pvt. Ltd., (India). Collagenase type IV and dispase were procured from SigmaAldrich (United States) and HiMedia Laboratories Pvt. Ltd., India respectively.

BD Vacutainer® PLUS plastic plasma blood collection tubes containing 143 USP units sodium heparin (spray-coated) anticoagulant were procured from Becton, Dickinson and Company (United States). HepG2 cell lines were procured from National Centre for Cell Science (India). For cell culture, Dulbecco's modified eagle's medium (DMEM) (Gibco, Invitrogen, United States) containing $2 \mathrm{mM}$ L-glutamine, supplemented with penicillin (100 units per $\mathrm{mL})$, streptomycin $\left(100 \mu \mathrm{g} \mathrm{mL}^{-1}\right)$

(HiMedia Laboratories Pvt. Ltd., India) and 10\% fetal calf serum (Invitrogen, United States) was used.

\subsection{Development of P HFMs}

P HFMs were developed as described in our previous study.,16 Briefly, $\mathrm{P}$ was vacuum dried in oven for $24 \mathrm{~h}$ at $80{ }^{\circ} \mathrm{C}$ to remove the absorbed moisture and dissolved in NMP to prepare polymer dope solution. The dope solution was degassed to remove dissolved gas from the solution. Indigenously fabricated HFM spinning pilot plant was used for developing HFMs. The process parameters used for HFM spinning are listed in Table 1. The prepared fibers were left in water for a day to remove the residual solvent and used for further studies.

\subsection{Surface functionalization of $P$ HFMs}

The surface functionalization of P HFMs was carried out in two steps: first step, amino groups were incorporated on outer surface of the PES HFMs, and second step, LBA were coupled with the animated P HFMs.

2.3.1. Amination of $\mathbf{P}$ HFMs. In order to incorporate amine groups onto the outer surface of P HFMs, reaction was started with chloromethylation as discussed in the literature. ${ }^{17}$ Briefly, the HFM samples were dipped in a solution of CMME, hexane and $\mathrm{SnCl}_{4}$ as the Friedel-Crafts catalyst at $25{ }^{\circ} \mathrm{C}$ for $10 \mathrm{~min}$. Reaction conditions were optimized to maintain the integrity of the membrane. $1.5 \mathrm{~mL}$ each of $\mathrm{SnCl}_{4}$ and chlorodimethyl ether (MCE) were added to the $10 \mathrm{~mL}$ hexane, and then PES HFMs were dipped in this solution for $10 \mathrm{~min}$. HFMs were rinsed by hexane to remove excess reactants left on surface of the HFM samples. Next, the HFM samples were dipped into $12 \mathrm{~mL}$ EDA for $20 \mathrm{~min}$. The reaction tube containing these HFM samples was placed in an ice bath during this process to counter the rise in temperature by the exothermic reaction.

2.3.2. LBA-functionalization of aminated HFMs. The surface of the aminated P HFMs was treated with EDC and NHS to attach LBA as discussed in the literature. ${ }^{18} 0.05 \mathrm{~g}$ LBA, $0.15 \mathrm{~g}$ NHS, $0.147 \mathrm{~g}$ EDC and $0.02 \mathrm{~g}$ MES were added to $1 \mathrm{~mL}$ Milli-Q water. The solution was left to rest for $15 \mathrm{~min}$ to allow for the formation of intermediates. This solution was then mixed with $10 \mathrm{~mL}$ 0.1 M PBS. The HFM samples were dipped in the resultant solution and left to rest for $36 \mathrm{~h}$. The samples were then taken out from the solution and thoroughly rinsed with Milli-Q to remove any solution residues. The prepared samples were

Table 1 Process parameters for spinning P HFMs

Ambient temperature $\left({ }^{\circ} \mathrm{C}\right)$

Relative humidity (\%)

Dope solution composition

Bore solution composition

Dope and bore solution temperature $\left({ }^{\circ} \mathrm{C}\right)$

Dope flow rate $\left(\mathrm{mL} \mathrm{min}{ }^{-1}\right)$

Bore flow rate $\left(\mathrm{mL} \mathrm{min}^{-1}\right)$

Air gap (cm)

Coagulation bath composition

Rinse bath composition

Coagulation bath temperature $\left({ }^{\circ} \mathrm{C}\right)$
25

50-60

$25 \mathrm{wt} \% \mathrm{P}$

Deionized (DI) water 25

2

2

45

DI water

DI water

25 


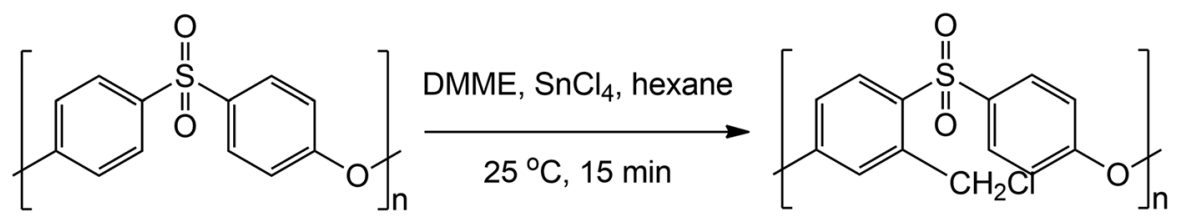

Polyethersulfone $(\mathrm{P})$

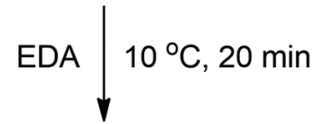<smiles></smiles>

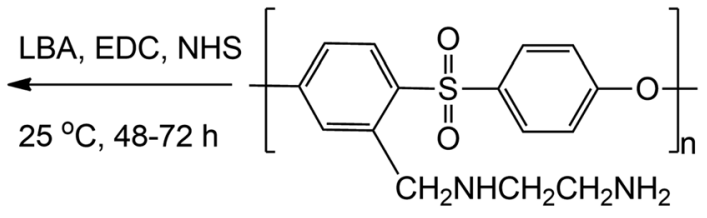

P-LBA

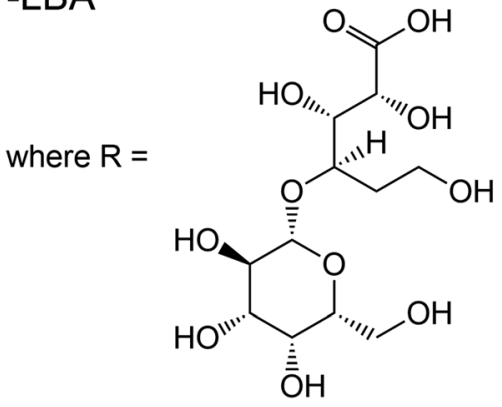

Fig. 1 Reaction scheme for surface functionalization of P HFMs resulting in P-LBA HFMs.

termed as P-LBA HFMs. Fig. 1 shows the sequence of steps to surface functionalize the P HFMs.

In this study, two samples were considered for the comparison purposes:

(1) P: plain HFMs.

(2) P-LBA: LBA-surface modified P HFMs.

\subsection{Characterization of HFMs}

The surface morphology of HFM samples (P and P-LBA) were observed by ESEM study. The HFM samples were then coated with gold/platinum by sputter coating using Auto fine coater JFC-1600 (JEOL, Japan) before observing under ESEM at 5-10 kV (Hitachi, S-3400 N, United Kingdom). EDS elemental mapping was also performed to map the elements present on the outer surface of P and P-LBA HFMs (X-Max $20 \mathrm{~mm}^{2}$ EDS system, Oxfords Instruments, United Kingdom). The surface modification of P-LBA HFMs was confirmed by ATR-FTIR (Bruker, Germany), and XPS (Kratos Analytical, United Kingdom) studies.

In addition to the above-mentioned characterization, the hydrophilicity and roughness of the biomaterial surface are also important factors, which influence the cell attachment, and protein adsorption. ${ }^{19}$ Water contact angles were measured using goniometer, Digidrop GBX (GBX Instruments, Romans, France) to assess the wettability of the HFM samples. Membrane surface roughness is another factor, which affects the attachment of the cells on the membrane surface. ${ }^{2021}$ The outer surface roughness was measured with the help of atomic force microscopy (AFM) (MFP-3 D-BIO, Asylum Research, USA). HFM samples were fixed on a cover slide with the help of adhesive tape. $5 \times 5 \mu \mathrm{m}$ area was scanned in the tapping mode in air. SiN probe cantilever having spring constant of $40 \mathrm{~N} \mathrm{~m}^{-1}$ was used for analysis. The average roughness $\left(R_{\mathrm{rms}}\right)$ of HFM samples was reported.

\subsection{Evaluation of compatibility of HFMs with human blood}

The compatibility of HFM samples with human blood was evaluated by measuring the hemolysis values. The protocol followed to perform hemolysis for HFM samples was described in the literature. ${ }^{21-23}$ Briefly, human blood was collected from a healthy donor in accordance with extant institutional guidelines and collected in BD Vacutainer ${ }^{\circledR}$ Plus plastic plasma tubes containing 143 USP units sodium heparin (spray-coated) anticoagulant. The collected blood sample was centrifuged at $1250 \mathrm{rpm}$ for $15 \mathrm{~min}$ at $10{ }^{\circ} \mathrm{C}$. After centrifugation, the supernatant containing platelet rich plasma was removed. The pellet containing erythrocytes were washed three times with normal saline solution (NSS) $(0.9 \% \mathrm{w} / \mathrm{v} \mathrm{NaCl})$. The obtained hematocrit was incubated with HFM samples (4 pieces of $2 \mathrm{~cm}$ length) for $1 \mathrm{~h}$ at $37{ }^{\circ} \mathrm{C}$ and $5 \%$ carbon dioxide $\left(\mathrm{CO}_{2}\right)$ in an incubator (Thermo Scientific ${ }^{\mathrm{TM}}$, United States). DI water and NSS were used as positive and negative controls, respectively. The samples were centrifuged at $1000 \mathrm{~g}$ for $5 \mathrm{~min}$. The red blood cells lysis was quantified using UV-visible spectrophotometer 


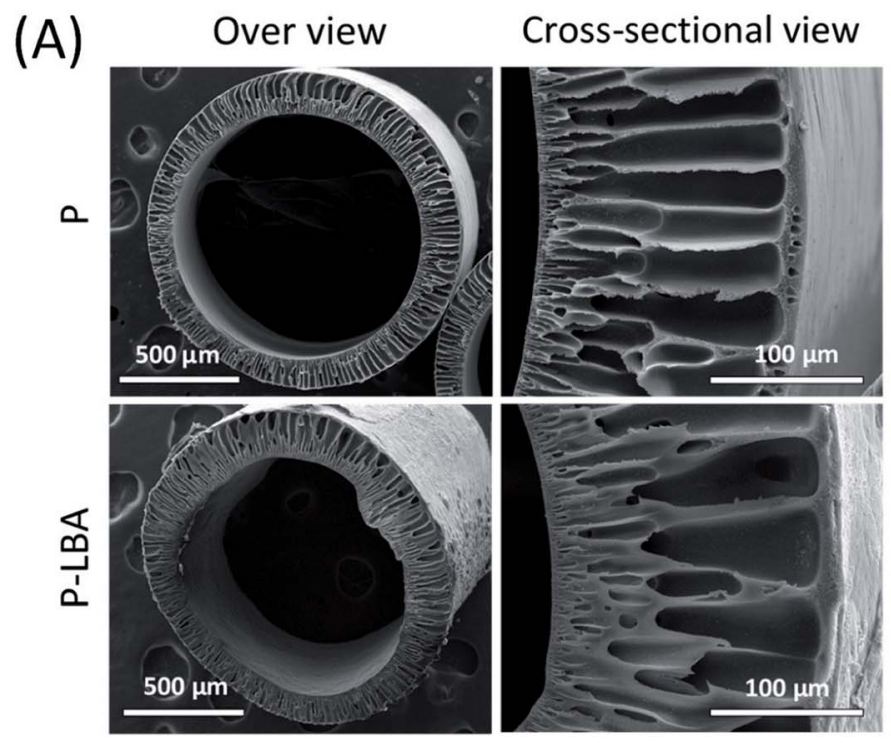

(B) PHFMs: $\mathrm{C}=68.50 \%, \mathrm{O}=\mathbf{2 0 . 0 3} \%, \mathrm{~S}=\mathbf{8 . 7 7} \%, \mathrm{~N}=\mathbf{2 . 7 0} \%$
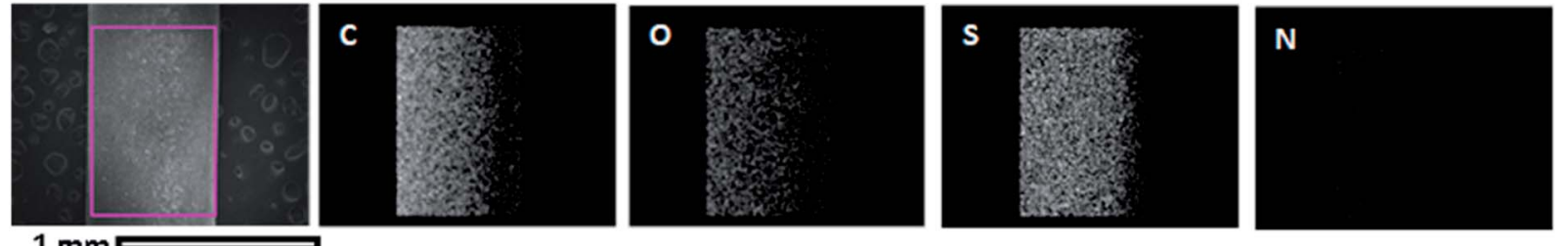

Aminated P HFMs: C $=46.21 \%, O=25.29 \%, S=3.33 \%, N=25.18 \%$
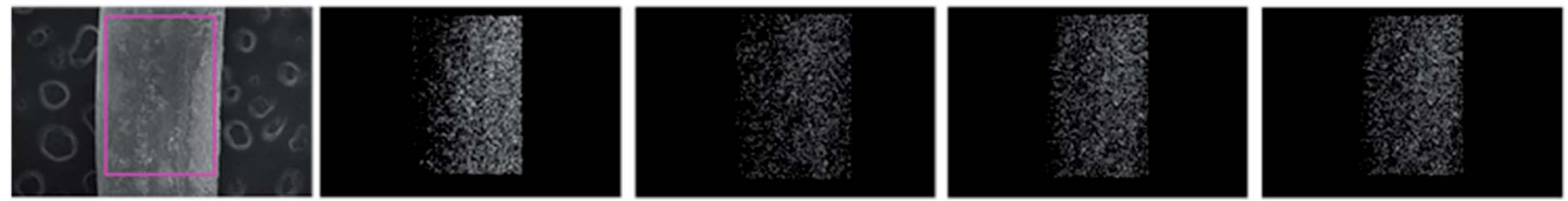

$1 \mathrm{~mm}$

P-LBA HFMs: $\mathrm{C}=57.27 \%, \mathrm{O}=23.75 \%, \mathrm{~S}=6.73 \%, \mathrm{~N}=12.25 \%$
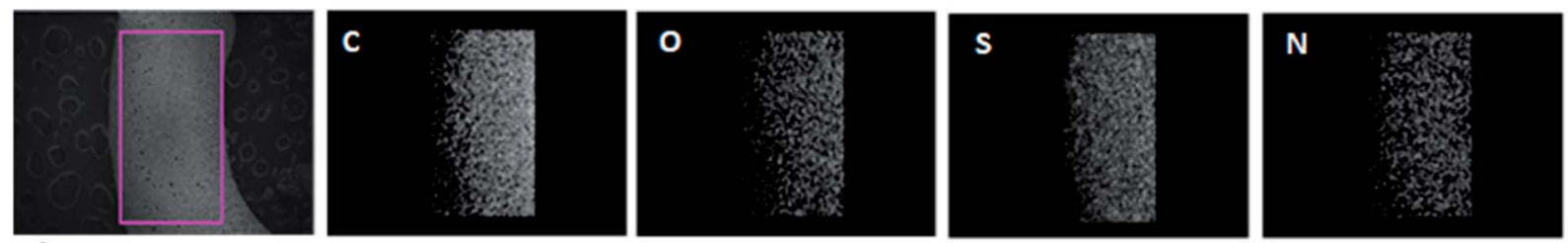

$1 \mathrm{~mm}$

Fig. 2 (A) ESEM micrographs of P and P-LBA HFMs showing the overview and cross-sectional view. Concentric HFMs with interconnected pores and fingers-like structures observed. (B) EDS elemental mapping of the outer surface of HFMs showed the presence of nitrogen in the P-LBA HFMs, which is an indicative of uniform surface modification via covalent coupling of LBA on aminated HFMs (P-LBA HFMs).

(Molecular Devices, United States) at $542 \mathrm{~nm}$. Hemolysis ratio (HR) was calculated using the equation: ${ }^{16}$

$$
\mathrm{HR}=\left(\frac{A_{\mathrm{S}}-A_{\mathrm{N}}}{A_{\mathrm{P}}-A_{\mathrm{N}}}\right) \times 100
$$

where $A_{\mathrm{S}}$ stands for the absorbance of sample supernatant, $A_{\mathrm{P}}$ and $A_{\mathrm{N}}$ are absorbance of positive and negative controls, respectively. 


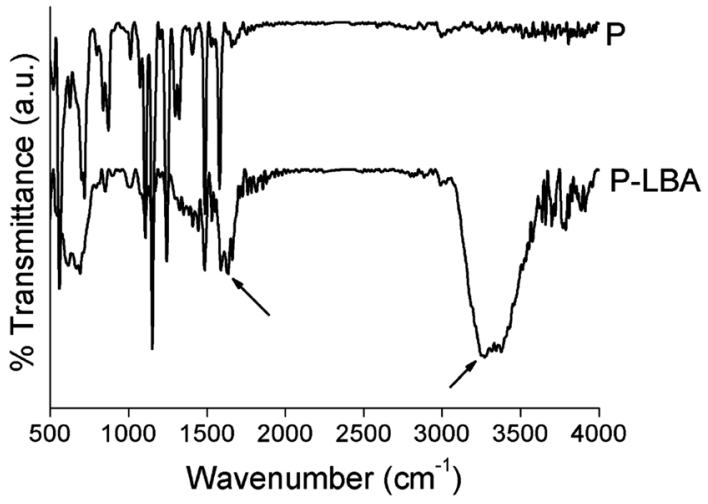

Fig. 3 ATR-FTIR spectra of $P$ and P-LBA HFMs. The presence of characteristic bands in the range $1580-1680 \mathrm{~cm}^{-1}$ corresponding to $-\mathrm{NH}_{2}$ and $-\mathrm{CO}-\mathrm{NH}$ - groups, and $3200-3400 \mathrm{~cm}^{-1}$ corresponding to $\mathrm{NH}$ and $-\mathrm{OH}$ groups confirmed the surface modification on HFMs.

\subsection{Study of human umbilical cord mesenchymal stem cells (hUMSCs) expansion on HFMs}

Obtaining an adequate number of cells from a donor for transplantation is a major bottleneck to the success of stem cell therapy. A substrate, which can support the growth and expansion of cell especially human stem cell, is always desired. $^{24,25}$ In this study, the HFM samples were studied for stem cell expansion. Human umbilical cords (hUCs) were obtained from a local maternity and health center after signing informed consents. UCs were collected in $50 \mathrm{~mL}$ falcon tube containing phosphate-buffered saline (PBS) supplemented with antibiotics, $100 \mathrm{U} \mathrm{mL}^{-1}$ penicillin and $100 \mu \mathrm{g} \mathrm{mL} \mathrm{m}^{-1}$ streptomycin and transported to the laboratory. ${ }^{26}$

2.6.1. Isolation of hUMSCs from umbilical cord tissue. The protocol for isolation of hUMSCs was adapted from a previous study. ${ }^{26}$ Briefly, the cord blood was removed from the cord by squeezing the cord by curved forceps. Blood clots were removed from blood vessels using sterile (PBS). Then after, the umbilical cord was processed for isolation of hUMSCs. Firstly, blood vessels from the UCs were removed by blunt dissection and remaining part of cord was chopped into fine pieces of 1-2 mm length using sterile surgical blade. The chopped tissues were

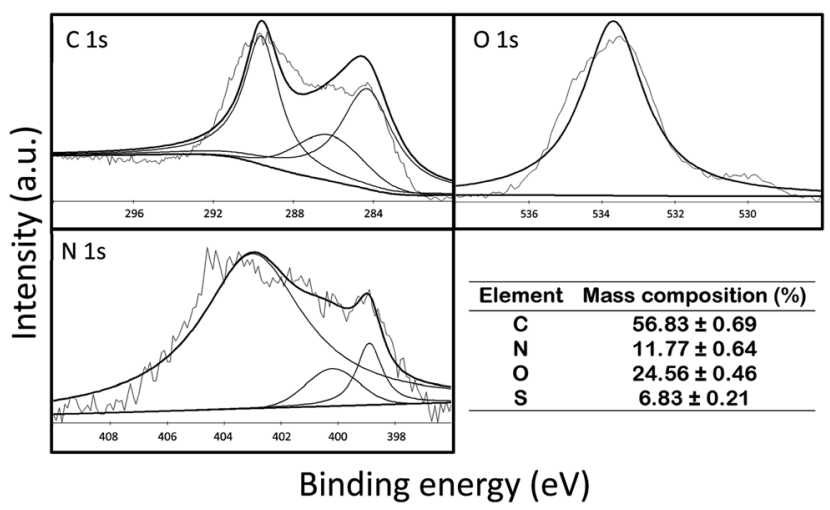

Fig. 4 XPS spectra for $C 1 s, O$ 1s and $N$ 1s for P-LBA HFMs. The appearance of the characteristic peaks confirmed the presence of amide and LBA linkages in the P-LBA HFMs. The mass composition (\%) is also shown alongside the XPS spectra. then subjected to enzymatic digestion for $30 \mathrm{~min}$ in an enzyme cocktail consisting of collagenase (type IV: dispase, $7: 1 \mathrm{v} / \mathrm{v}$ ) on a magnetic orbital shaker at $37^{\circ} \mathrm{C}$. Further, a mixture of trypsin (0.05 wt \%) and EDTA (0.02 wt\%) was added and incubated on a magnetic orbital shaker for $15-20 \mathrm{~min}$ at $37^{\circ} \mathrm{C}$. The digested tissues were then filtered using sterile nylon filter and centrifuged at $1500 \mathrm{rpm}$ for $10 \mathrm{~min}$. Finally, the pellet was resuspended, cultured in DMEM and incubated at $37{ }^{\circ} \mathrm{C}$ and $5 \% \mathrm{CO}_{2}$. The culture media were replaced every two days alternatively.

2.6.2. Characterization of isolated hUMSCs. Upscaled hUMSCs at passage 3 were seeded on cover slip. Next they were fixed in $4 \%$ paraformaldehyde for $15 \mathrm{~min}$ at $4{ }^{\circ} \mathrm{C}$ followed by permeabilization in $0.2 \%$ Triton X-100. After blocking in bovine serum (HiMedia Laboratories Pvt. Ltd., India), the cells were washed gently. Primary antibodies, anti-Oct-4 (Biolegend), and FITC mouse anti-human CD90 (Biolegend) were incubated with cells for $2 \mathrm{~h}$. After washing with PBS solution, a secondary antibody, namely, Alexa fluor 488 goat anti mouse IgG (Invitrogen, Carlsbad, CA, United States), was added. Cover slip was mounted, and fluorescent images were captured using spinning confocal microscope (Zeiss, Germany).

2.6.3. Seeding of hUMSCs on HFMs. hUMSCs were cultured in DMEM at $37{ }^{\circ} \mathrm{C}$ in $\mathrm{CO}_{2}$ incubator. HFM samples with equal surface area, were used for cell seeding. Firstly, samples were sterilized by dipping in $70 \%$ ethanol followed by exposing to UV-light for $30 \mathrm{~min}$. Excess of ethanol was removed by washing thoroughly with sterile PBS. $1 \times 10^{3}$ cells were seeded on $1 \mathrm{~cm}^{2}$ HFM samples at passage three. Cells were allowed to attach with these samples for $4 \mathrm{~h}$, and then the HFM samples with cells were transferred into 12-well plate with fresh culture medium.

At day 3 and 6, the samples were gently washed with PBS. The HFM samples with cells were evaluated for nutrient consumption and cell proliferation.

2.6.3.1. hUMSCs viability and proliferation on HFMs. 3(4,5-Dimethylthiazol-2-yl)-2,5-diphenyltetrazoliumbromide (MTT) cell viability assay was performed to evaluate viability of hUMSCs on P and P-LBA HFM samples after day 3 and day 6 of incubation. $500 \mu \mathrm{L}$ of MTT was added to each well. After $4 \mathrm{~h}$ incubation at $37{ }^{\circ} \mathrm{C}, 500 \mu \mathrm{L}$ dimethyl sulfoxide (DMSO) was added in each sample to dissolve the formazan crystals. Color

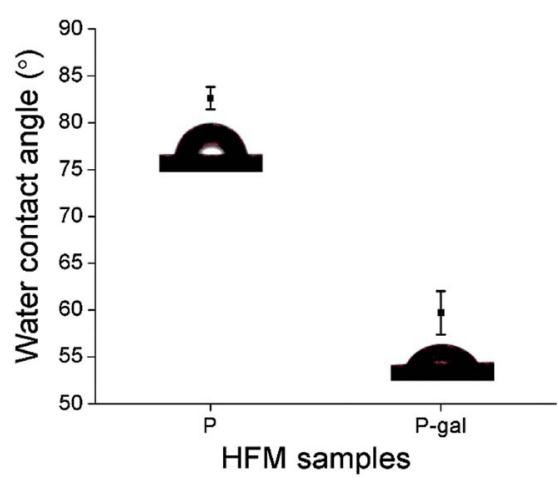

Fig. 5 Water contact angles of P and P-LBA HFMs. Improvement in hydrophilicity of HFMs achieved with the surface modification. 


\section{$\mathrm{P} \sim \mathrm{R}_{\mathrm{RMS}}=9.4 \pm 1.8 \mathrm{~nm}$}
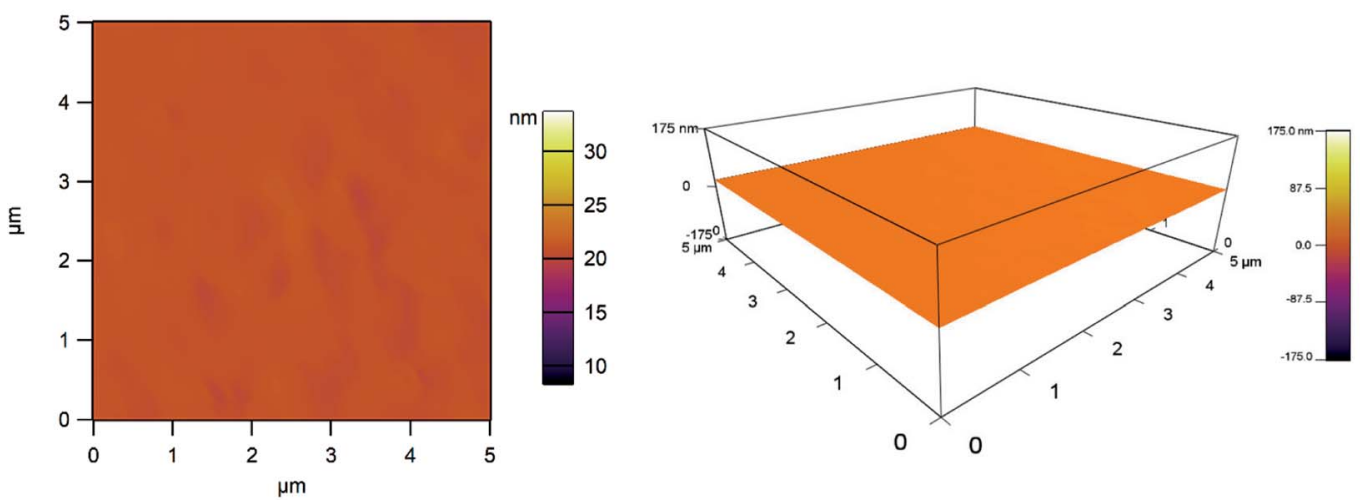

$$
\mathrm{P}-\mathrm{LBA} \sim \mathrm{R}_{\mathrm{RMS}}=33.9 \pm 2.1 \mathrm{~nm}
$$
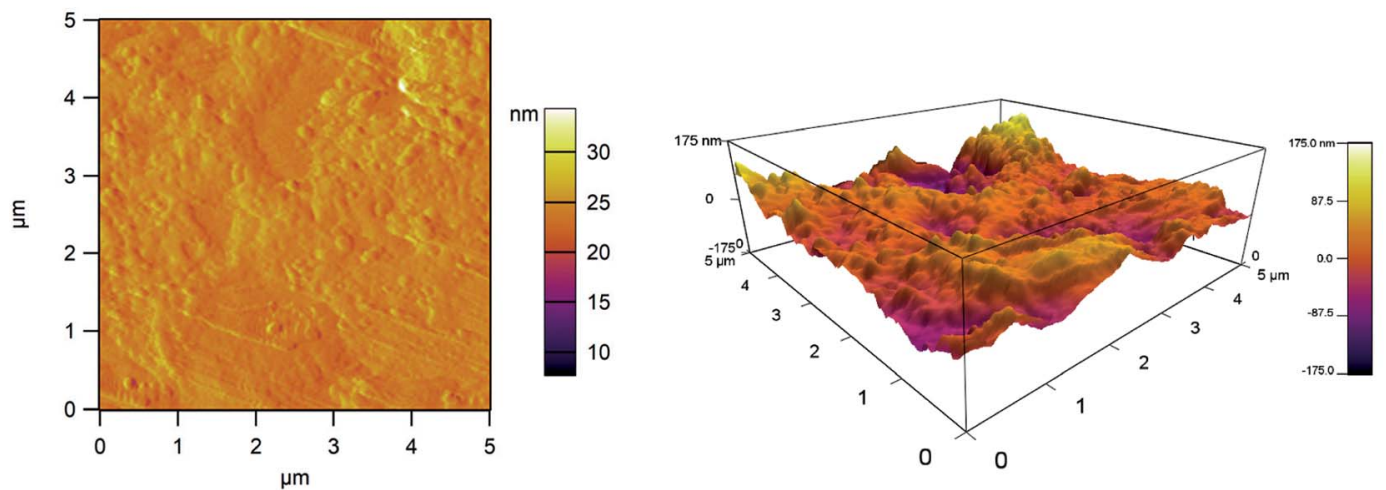

Fig. 6 AFM images of P and P-LBA HFMs. The increased roughness on the outer surface of P-LBA HFMs enhanced the attachment of HepG2 cells.

change was observed as, yellow color of MTT was reduced to purple color of formazan in presence of living cells. The optical density of the formazan solution was determined by a UV visible spectrophotometer (Molecular Devices, United States) at $560 \mathrm{~nm}$.

The quantitative evaluation of cell proliferation on HFM was assessed using Quant-iT PicoGreen ds DNA assay kit (Molecular probe, Invitrogen, United States). The HFM, seeded with cells were harvested at set time points. The samples were washed with PBS and dipped in deionized water to lyse the cells. Thereafter, the samples were frozen and thawed alternatively twice and centrifuged at $10000 \mathrm{rpm}$ for $10 \mathrm{~min}$. Then, $100 \mu \mathrm{L}$ of PicoGreen working solution was added to supernatant of each sample, and left for incubation for $5 \mathrm{~min}$. The concentration of DNA was measured using a fluorescence microplate reader (Spectramax M2e. Molecular devices, United States). The fluorescence readings were recorded at $480 \mathrm{~nm}$ excitation and $538 \mathrm{~nm}$ emission. The DNA amount was calculated by using the standard curve as prescribed by the manufacturers' kit. The HFM samples with cells were also evaluated for nutrient consumption (by determining the glucose levels in the culture media of different HFMs).

\subsection{HepG2 cell culture study}

HepG2 cells were cultured in DMEM containing $2 \mathrm{mM} \mathrm{L}$ glutamine, supplemented with penicillin (100 units per $\mathrm{mL}$ ), streptomycin $\left(100 \mathrm{mg} \mathrm{mL}^{-1}\right)$ and $10 \%$ fetal calf serum at $37^{\circ} \mathrm{C}$ in $\mathrm{CO}_{2}$ incubator. HFM samples with equal surface area, were used for cell seeding. Samples were sterilized by dipping in $70 \%$ ethanol followed by exposing to UV-light for $30 \mathrm{~min}$. Excess of ethanol was removed by washing thoroughly with sterile PBS. $1 \times 10^{3}$ cells per $\mathrm{cm}^{2}$ were seeded on HFM samples. Cells were allowed to attach with these samples for $4 \mathrm{~h}$, and then the HFM samples with cells were transferred into 12-well plate with fresh culture medium. ${ }^{21}$ At day 3 and 6 , the samples were gently washed with PBS. The HFM samples with cells were evaluated for nutrient consumption, cell proliferation with characteristic morphology. HepG2 cell functional study was performed by evaluating the urea synthesis, albumin secretion and glucose consumption in the culture media.

2.7.1. Cell morphology and proliferation study. At predetermined set point of each of these studies, the attached cells were observed by confocal microscopy (Zeiss LSM 780, Germany). The samples were prepared and observed as discussed in previous studies., 


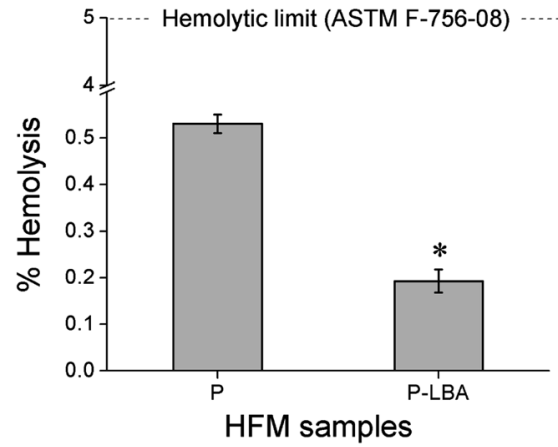

Fig. 7 Bar charts showing \% hemolysis values of $\mathrm{P}$ and P-LBA HFMs. Both fall within the hemolytic limit $(5 \%)$ indicating the suitability for blood-contacting applications.

3-(4,5-Dimethylthiazol-2-yl)-2,5-diphenyltetrazoliumbromide (MTT)-based cytotoxicity test was performed to evaluate cytocompatibility of the HFM samples. After incubation of day 3 and day 6, $500 \mu \mathrm{L}$ MTT was added to each well. After $4 \mathrm{~h}$ incubation at $37^{\circ} \mathrm{C}, 500 \mu \mathrm{L}$ dimethyl sulfoxide (DMSO) was added to each well to dissolve the formazan crystals. The optical density of the formazan solution was determined by a UV-visible spectrophotometer (Molecular Devices, United States) at $560 \mathrm{~nm}$. In addition to this, DNA quantification was performed as described in the earlier Section 2.6.3.1.

2.7.2. Cell function study. The cells function studies were performed at day 3 and day 6 . The studies include urea synthesis, albumin secretion and glucose consumption.

Urea synthesis and albumin secretion are the liver cell specific functional marker. ${ }^{27}$ Spent cell culture media were evaluated for urea and albumin concentration at day 3 and day 6. The detection procedure was followed as per the estimation kit manufacturers' protocols. The glucose concentration in the spent medium was determined with a biochemical GOD/POD method using a kit procured from Coral Clinical Systems (India). In this, glucose is oxidised to gluconic acid and hydrogen peroxide in the presence of glucose oxidase. Further, hydrogen peroxide reacts with phenol and 4-aminoantipyrine by the catalytic action of peroxidase to form a red coloured quinoneimine dye complex. The glucose consumption was calculated at day 3 and day 6 . This help to evaluate cell function and continuous proliferation.

\section{Results and discussion}

In this section, the results related to the physicochemical characterization, biocompatibility including hemocompatibility evaluation, and HepG2 functions are presented and discussed.

\subsection{HFM characterization}

In this section, the results discussing the physicochemical characterization of HFMs by ESEM, EDS elemental mapping, ATR-FTIR, XPS, water contact angle, and AFM are presented.

3.1.1. Surface morphology by ESEM. The developed HFMs were characterized for morphology using ESEM. Fig. 2A shows the overview and cross-sectional view of the HFM samples. Overview shows the concentricity of the HFMs. In addition to this, images of the cross sectional view of HFM samples exhibited the interconnected finger-like pores.

Fig. 2B shows the EDS elemental mapping of both the HFM samples: $\mathrm{P}$ and P-LBA. The elemental map of $\mathrm{N}$ in P-LBA HFMs clearly indicates that the surface modification of HFMs was uniform across the length and width of the HFMs. Further, the change in intensity from left to right of an elemental map is due to the curvature of the HFMs.

3.1.2. Confirmation of surface modification of HFMs by ATR-FTIR analysis. ATR-FTIR analysis was performed to confirm the chemical modification of HFMs (Fig. 3). The FTIR spectra of $\mathrm{P}$ and P-LBA HFMs were compared. The characteristic peaks of $\mathrm{P}$ were recorded as discussed in previous study. ${ }^{21}$ However, with the surface modification of HFMs, the change in
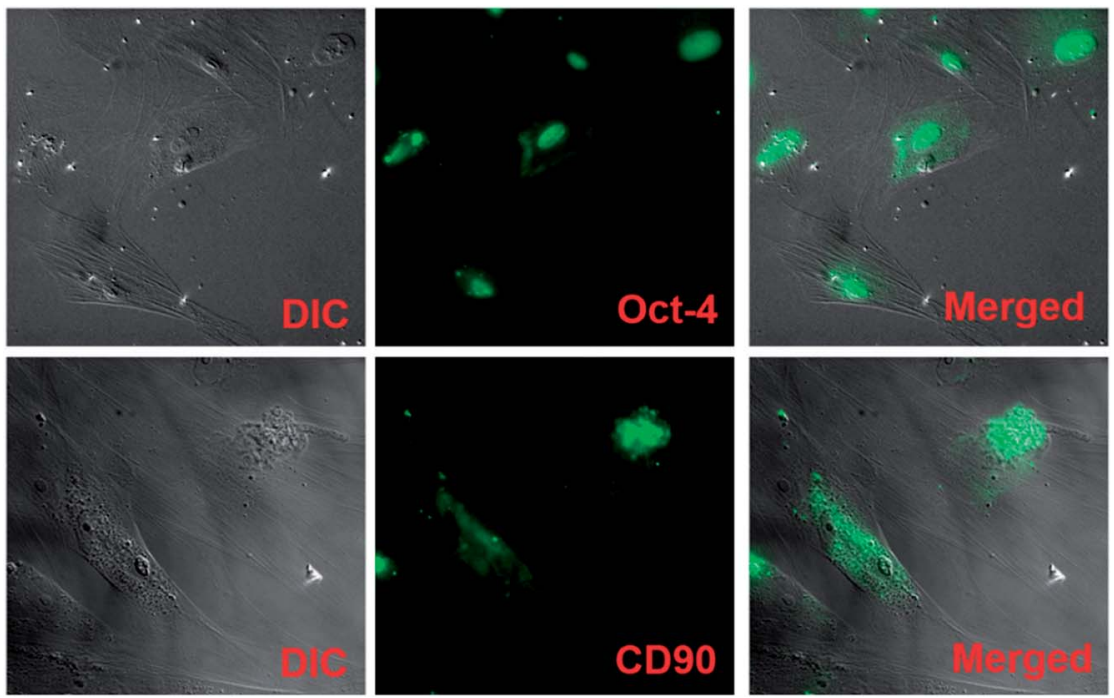

Fig. 8 hUMSC characterization: DIC images show the fibroblast-like morphology of hUMSCs. Mesenchymal stem cell markers, Oct-4 and CD90, exhibit immunofluorescence in green color. Merged images show hUMSCs with fluorescence. 
intensity of these peaks was recorded. Further, the appearance of peaks in the range $1580-1680 \mathrm{~cm}^{-1}$ corresponding to $-\mathrm{NH}_{2}$ and - $\mathrm{CO}-\mathrm{NH}-$ groups was recorded, which indicated the attachment of EDA to LBA. ${ }^{28}$ In addition to this, the characteristic band in the range $3200-3400 \mathrm{~cm}^{-1}$ corresponding to $\mathrm{NH}$ and $-\mathrm{OH}$ groups was also recorded in the spectrum, which indicated the presence of LBA on the aminated outer surface of P-LBA HFMs.

3.1.3. X-ray photoelectron spectroscopy. Fig. 4 represents the XPS spectra for C 1s, O 1 s and $\mathrm{N}$ 1s for P-LBA HFMs. For C 1s, the peaks appearing at $284.3 \mathrm{eV}, 286.2 \mathrm{eV}$, and $289.6 \mathrm{eV}$ corresponded to $\mathrm{C}-\mathrm{C} / \mathrm{C}-\mathrm{H}$ bond, $\mathrm{C}-\mathrm{O}$ bond, and $\mathrm{C}=\mathrm{O}$ bond, respectively. For $\mathrm{O}$ 1s, the two peaks appeared at $532.5 \mathrm{eV}$ and $534.3 \mathrm{eV}$, which represented $\mathrm{C}=\mathrm{O}$ and $\mathrm{C}-\mathrm{O}-\mathrm{C} / \mathrm{C}-\mathrm{OH}$ bonds, respectively. For N 1s, the peaks were recorded at 398.9, 400.2, and $403 \mathrm{eV}$, which corresponded to pyridinic, pyrrolic, and graphitic bonds, respectively. Furthermore, the mass composition (\%) of C, N, O and S in P-LBA HFMs was $56.83 \pm 0.69,11.77$ $\pm 0.64,24.56 \pm 0.46$, and $6.83 \pm 0.21$. Thus, the XPS results clearly confirmed the presence of amide and LBA linkages in the P-LBA HFMs as discussed in the earlier results.

3.1.4. Water contact angle. The hydrophilicity of the P-LBA HFMs was found to significantly enhance with respect to pristine P HFMs. The water contact angle decreased from $82.6 \pm$ $1.2^{\circ}$ for P HFMs to $59.7 \pm 2.3^{\circ}$ for P-LBA HFMs (Fig. 5). Several literature studies showed that the improved hydrophilicity either by blending hydrophilic additive to the native $\operatorname{PES}^{\mathbf{1 6 , 2 1 , 2 2 , 2 9}}$ or chemically modifying the membrane $e^{23,30,31}$ resulted in enhanced cell adhesion. The positive effect of improving hydrophilicity on cell attachment and proliferation was observed in this study, which is discussed later in the manuscript.

3.1.5. Surface roughness by AFM. Topographies of both native $\mathrm{P}$ and P-LBA HFMs were observed by AFM, as shown in Fig. $6\left(R_{\text {rms }}\right.$ values are reported). The roughness value of the PLBA HFMs $(33.9 \pm 2.1 \mathrm{~nm})$ increased as compared to that of $P$ HFMs $(9.4 \pm 1.8 \mathrm{~nm})$. The covalently attached LBA increased the outer surface roughness, which helped to attach more cells on the surface, ${ }^{9,13,23}$ as discussed later in the manuscript.

\subsection{Biocompatibility evaluation of the developed HFMs}

The HFM samples were evaluated for hemocompatibility and cytotoxicity. The related results are presented and discussed here.

3.2.1. Hemocompatibility evaluation: hemolysis. Hemolysis ratio (HR) is also used to evaluate the blood compatibility with a biomaterial. According to the ASTM F-756-08 standard, biomaterial having hemolysis less than $5 \%$ is suitable for applications involving human blood. P-LBA HFMs showed lower hemolysis ratio as compared to plain P HFMs. Hydrophilic surface modification improves surface
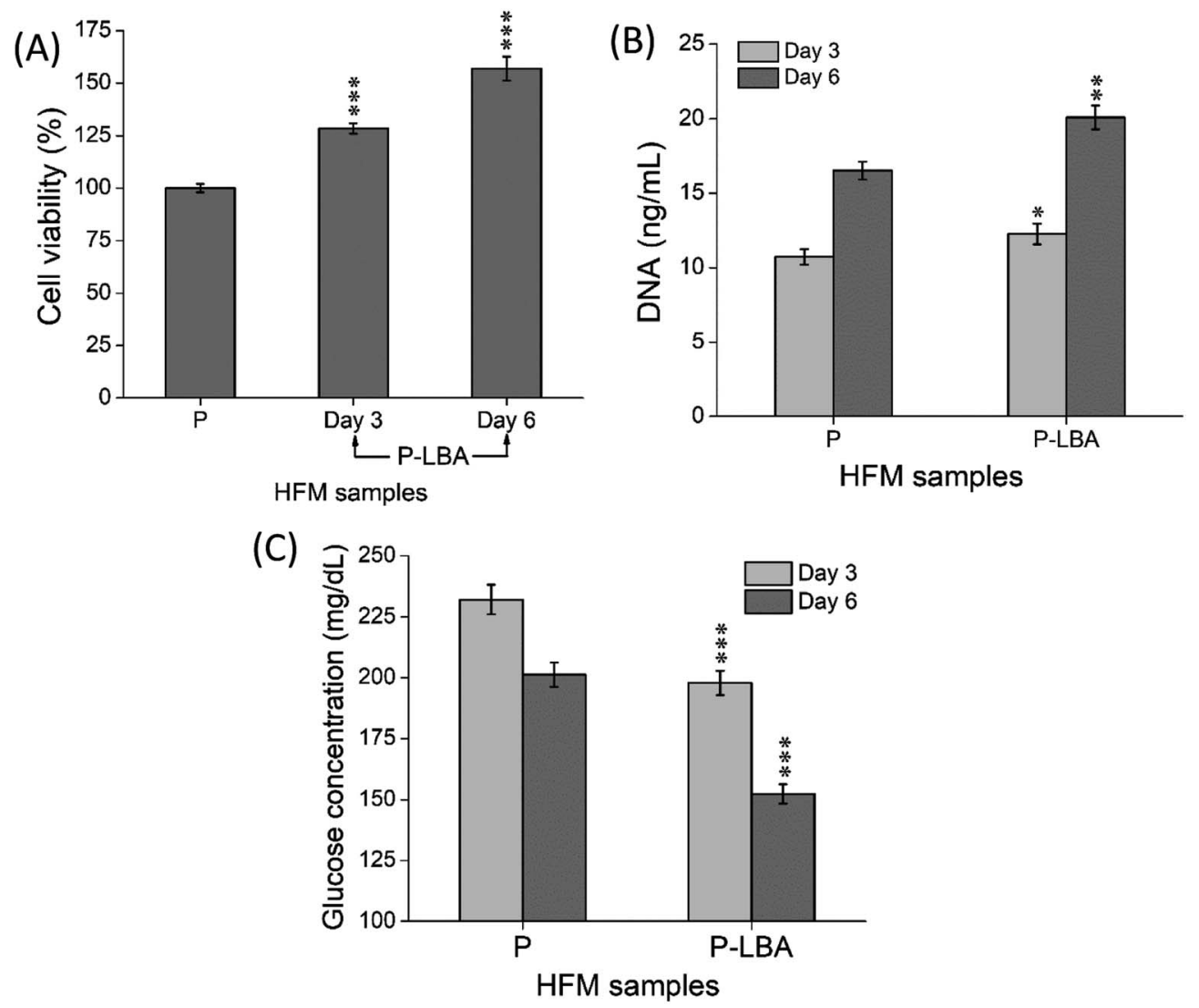

Fig. 9 Quantitative evaluation of (A) \% viability and (B) DNA content of cells attached on different HFMs. (C) Glucose levels in the spent cell culture media of different HFMs. The results indicated the suitability of P-LBA HFMs for the hUMCSs attachment and proliferation. Values are expressed as mean \pm SD $(n=3) ; * p<0.05, * * p<0.01, * * * p<0.001$ versus P HFMs. 

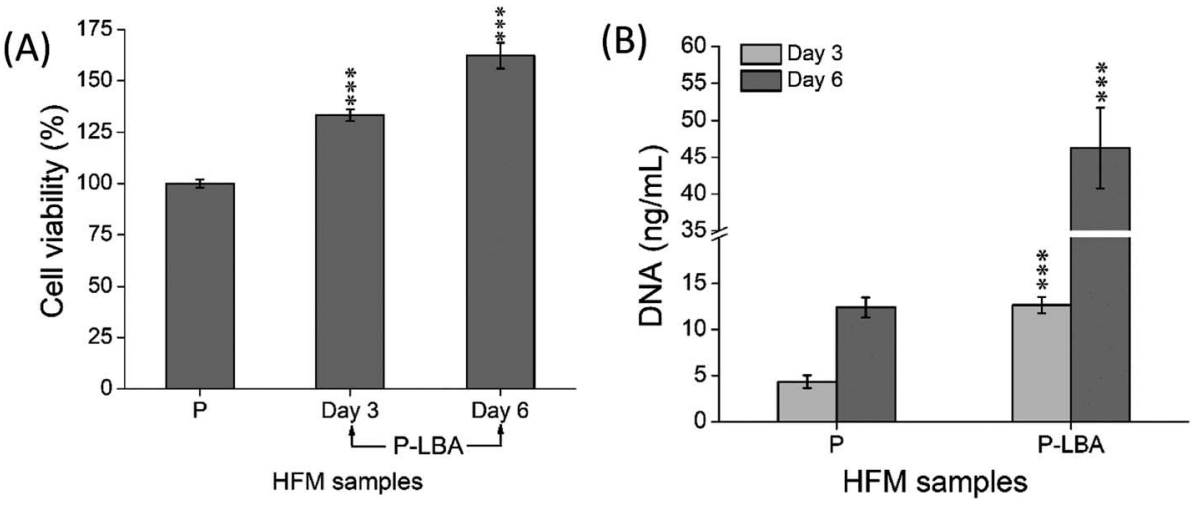

Fig. 10 Quantitative evaluation of (A) \% viability and (B) DNA content of HepG2 cells attached on different HFMs. The results indicated the suitability of P-LBA HFMs for the cell attachment and proliferation. Values are expressed as mean \pm SD $(n=3) ; * * * p<0.001$ versus P HFMs.

hemocompatibility. ${ }^{32}$ Both the samples fall under the nonhemolytic limit as shown in Fig. 7. Thus, P and P-LBA HFMs are suitable for blood contacting applications.

3.2.2. Characterization of isolated hUMSCs. Mesenchymal stem cell markers were observed by immunofluorescence staining. Oct-4 (embryonic marker) expression was observed as secondary antibody emitted green fluorescence for Oct-4 marker. ${ }^{26} \mathrm{CD} 90$, a cell surface marker was observed separately by FITC conjugated $\mathrm{I}^{\mathrm{O}}$ antibody. ${ }^{33}$ Immunofluorescence and morphological analysis confirms the presence of hUMSCs (Fig. 8).

3.2.2.1. hUMSCs viability and proliferation on HFMs. MTT and PicoGreen assays were performed at the set time points. MTT assay depicted the enhanced cell viability form day 3 to day 6 (Fig. 9A). PicoGreen double stranded DNA (dsDNA) reagent is an ultra-sensitive fluorescent nucleic acid stain for dsDNA quantification. PicoGreen assay showed the increased DNA amount with P-LBA HFMs, which confirmed the cell proliferation on HFMs (Fig. 9B). DNA content was measured to further increase in day 6 as compared to day 3 for both the HFM samples. The amount of DNA is proportional to cellular growth. Cell proliferation was observed more on HFMs, modified with LBA.

Furthermore, glucose levels were also measured in the culture media of different HFMs, which are shown in Fig. 9C. In general, the lower glucose levels in the spent culture medium are desirable. In this study, the glucose levels associated with PLBA HFMs were found to be lower than that of the P HFMs on both the set time points. Thus, the results of MTT cell viability, PicoGreen DNA quantification, and glucose consumption assays confirmed the increase in the cell viability and proliferation.

\subsection{HepG2 cell culture study}

It has been known that apart from energy source, carbohydrates can serve as structural components of the natural product as a key element in various molecular recognition processes, including bacterial and viral infections, cell adhesion, differentiation, development, regulation and signal transduction events. ${ }^{\mathbf{8}, 34}$ Carbohydrate recognizing receptors found on the cell surface including hepatocytes, alveolar macrophages, and L1210 mouse leukemia cell are specifically bound to galactose/ $N$-acetyl- $b$-galactosamine, ${ }^{35}$ a-mannose ${ }^{36}$ and a-fucose, ${ }^{37}$ respectively. ASGPR recognizes galactose as a specific ligand, which was discovered and characterized in mammalian liver. ${ }^{38}$ In this part of study, HepG2 cell adhesion, proliferation and morphology study were performed with the help of confocal microscopy. The results of the cell function studies were also presented and discussed.

3.3.1. Cytotoxicity study. To examine the growth and proliferation of HepG2 cells on HFM samples, MTT assay was performed at day 3 and day 6. More cell proliferation was observed on the P-LBA HFMs as compared to P HFMs (Fig. 10A). In addition to this, the amount of DNA for the modified P-LBA HFMs was found to be more than that of the P HFMs (Fig. 10B), which clearly indicated the suitability of P-LBA HFMs for the cell attachment and proliferation. The surface modification of

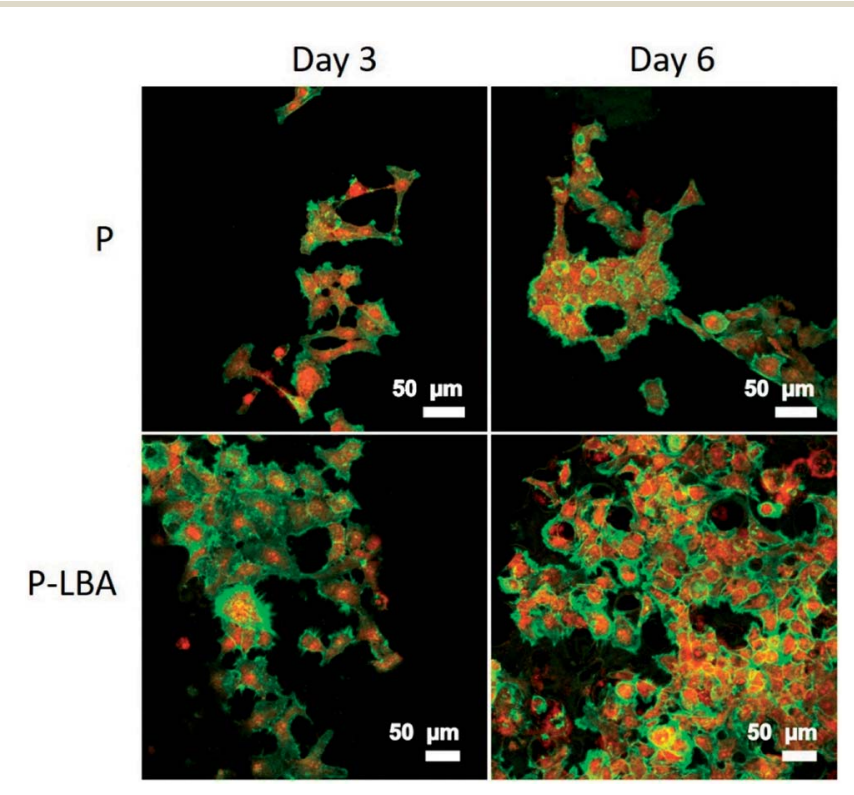

Fig. 11 Confocal micrographs showing HepG2 cell growth on $\mathrm{P}$ and P-LBA HFMs: enhanced HepG2 cell attachment and proliferation observed on P-LBA HFMs. Nuclei was stained with PI (red), and actins with FITC-ph (green). 
(A)

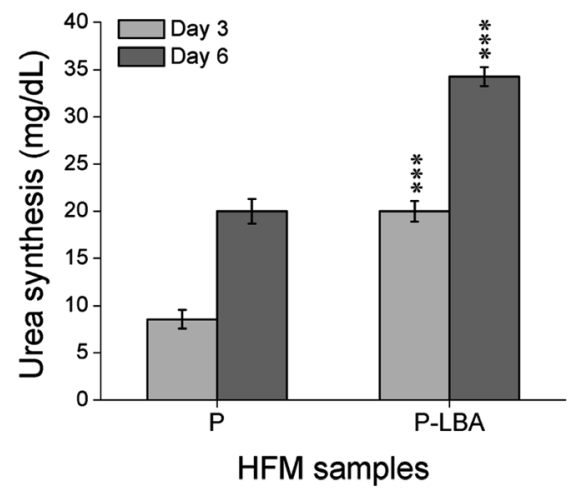

(B)

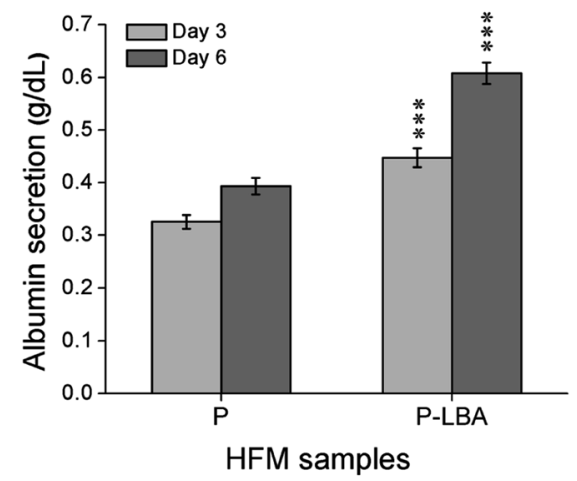

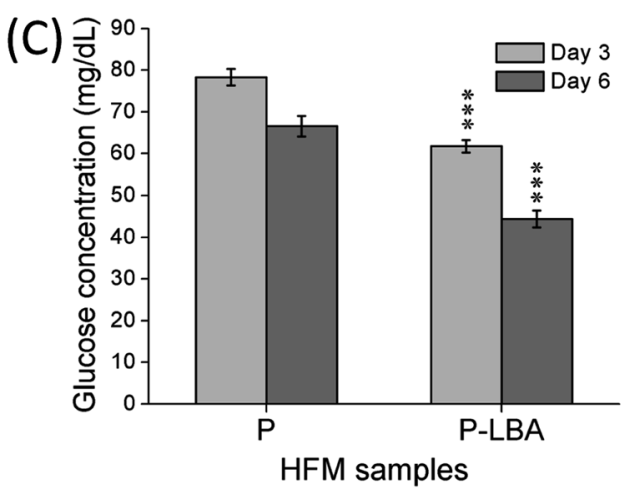

Fig. 12 Quantitative determination of (A) urea synthesis, (B) albumin secretion, and (C) glucose consumption in the spent medium of HFM samples (P and P-LBA) at day 3 and day 6 . Higher values of urea and albumin, and lower value of glucose on both the day were measured with $P$ LBA HFMs, which confirmed the high attachment and proliferation of cells on P-LBA HFMs as observed in the microscopy studies. Values are expressed as mean $\pm \mathrm{SD}(n=3) ; * * * p<0.001$ versus P HFMs.

HFMs with ECM like material promote the HepG2 cell attachment and proliferation. ${ }^{3}$

3.3.2. Confocal microscopy study. The morphology of the HepG2 cells attached on the outer surface of the HFM samples was observed at day 3 and day 6 (Fig. 11). Multicellular aggregates of HepG2 cells were observed on HFMs as reported in the literature. ${ }^{39}$ P-LBA HFMs showed enhanced cell growth, which can be corroborated with the cell viability assay. This study indicated that P-LBA HFMs supported the adherence and growth of HepG2 cells.

3.3.3. Cell functional study. Cell functional studies including urea synthesis, albumin secretion and glucose consumption were performed to evaluate the efficacy of the HFM samples.

Urea synthesis and albumin secretion are the key markers of HepG2 liver cell functional evaluation, which was measured in the spent media, on days 3, and 6. Enhanced urea and albumin was quantified with P-LBA HFMs (Fig. 12A and B). The binding of galactosylated alginate with hepatocytes via the patch of ASGPR improved liver functions of hepatocytes. ${ }^{13}$ Furthermore, glucose is the primary source of energy in living systems. It is an important factor for cells growth and viability. ${ }^{40}$ High glucose consumption was measured with P-LBA HFMs (Fig. 12C). LBAmodification promoted more cells to attach on the outer surface of P-LBA HFMs, resulting in higher consumption of glucose as compared to P-HFMs.

\section{Conclusions}

In this study, LBA was covalently coupled with P HFMs. First of all, P HFMs were prepared by phase inversion process with the help of our indigenously developed HFM spinning pilot plant. The outer surface of HFMs was modified with the LBA as a ASGPR ligand. The functional modification was confirmed by ATR-FTIR, EDS elemental mapping, and XPS analysis. The surface modification improved hydrophilicity as exhibited by water contact angle measurements, and also surface roughness, which is desirable for cells attachment and proliferation. P-LBA HFMs were found to be homocompatible with human blood as evaluated by hemolysis. Cytocompatibility was evaluated with human primary mesenchymal stem cells and HepG2 liver cells, which was found to be improved. Enhanced attachment and proliferation of both the cells was observed. The urea synthesis, albumin secretion and glucose consumption analysis showed the active functionality of the attached HepG2 cells on the PLBA HFMs. Thus, in this study, the P-LBA HFMs, developed with LBA-modification, may open up the possibility to use it for various applications including (1) HFM membrane material for bio-artificial liver development, (2) stem cell expansion, (3) drug testing bioreactor, and (4) mass cell culture. 


\section{Conflicts of interest}

There are no conflicts to declare.

\section{Acknowledgements}

The authors acknowledge the help of Dr Rohit Teotia in the hUMSCs culture studies. The authors are thankful to Centre for Research in Nanotechnology and Science (CRNTS), National Centre for Photovoltaic Research and Education (NCPRE), and Central Instrumentation Facility, Indian Institute of Technology Bombay, India for providing the access to various characterization facilities used in this study. The authors acknowledge research fellowship grants from the U.G.C. (S. K. V.), M.H.R.D. (A. M). The authors also acknowledge the Wadhwani Research Centre for Bioengineering, IIT Bombay, India for the research grants.

\section{References}

1 R. Unger, Q. Huang, K. Peters, D. Protzer, D. Paul and C. Kirkpatrick, Biomaterials, 2005, 26, 1877-1884.

2 K.-V. Peinemann and S. P. Nunes, Membrane Technology, Volume 1: Membranes for Life Sciences, John Wiley \& Sons, 2011.

3 S. K. Verma, A. Modi and J. Bellare, Biomater. Sci., 2018, 6, 280-291.

4 S. K. Verma, A. Modi, A. K. Singh, R. Teotia and J. Bellare, J. Biomed. Mater. Res., Part B, 2018, 106, 1286-1298.

5 B. Su, S. Sun and C. Zhao, in Progress in Hemodialysis-From Emergent Biotechnology to Clinical Practice, InTech, 2011.

6 J. M. Anderson, Annu. Rev. Mater. Res., 2001, 31, 81-110.

7 B. D. Ratner, J. Biomed. Mater. Res., Part A, 1993, 27, 837-850.

8 C. Cho, S. Seo, I. Park, S. Kim, T. Kim, T. Hoshiba, I. Harada and T. Akaike, Biomaterials, 2006, 27, 576-585.

9 R. S. Teotia, D. Kalita, A. K. Singh, S. K. Verma, S. S. Kadam and J. R. Bellare, ACS Biomater. Sci. Eng., 2015, 1, 372-381.

10 S. K. Verma, A. Modi, A. K. Singh, R. Teotia, S. Kadam and J. Bellare, Colloids Surf., B, 2018, 164, 358-369.

11 H.-F. Lu, W. S. Lim, J. Wang, Z.-Q. Tang, P.-C. Zhang, K. W. Leong, S. M. Chia, H. Yu and H.-Q. Mao, Biomaterials, 2003, 24, 4893-4903.

12 E. F. Neufeld and G. Ashwell, in The biochemistry of glycoproteins and proteoglycans, Springer, 1980, pp. 241-266.

13 J. Yang, M. Goto, H. Ise, C.-S. Cho and T. Akaike, Biomaterials, 2002, 23, 471-479.

14 H. K. Kleinman, R. J. Klebe and G. R. Martin, J. Cell Biol., 1981, 88, 473-485.

15 J. Lee, M. J. Cuddihy and N. A. Kotov, Tissue Eng., Part B, 2008, 14, 61-86.

16 G. J. Dahe, R. S. Teotia, S. S. Kadam and J. R. Bellare, Biomaterials, 2011, 32, 352-365.
17 A. Higuchi, K. Shirano, M. Harashima, B. O. Yoon, M. Hara, M. Hattori and K. Imamura, Biomaterials, 2002, 23, 26592666.

18 S. K. Vashist, Diagnostics, 2012, 2, 23-33.

19 D. Vaikkath, R. Anitha, B. Sumathy and P. D. Nair, Colloids Surf., B, 2016, 141, 112-119.

20 T.-W. Chung, D.-Z. Liu, S.-Y. Wang and S.-S. Wang, Biomaterials, 2003, 24, 4655-4661.

21 A. Modi, S. K. Verma and J. Bellare, J. Colloid Interface Sci., 2017, 504, 86-100.

22 A. Modi, S. K. Verma and J. Bellare, Mater. Sci. Eng., C, 2018, 91, 524-540.

23 A. Modi, S. K. Verma and J. Bellare, Colloids Surf., B, 2018, 167, 457-467.

24 O. Lindvall, Z. Kokaia and A. Martinez-Serrano, Nat. Med., 2004, 10, S42.

25 L. T. Lock and E. S. Tzanakakis, Tissue Eng., 2007, 13, 13991412.

26 R. S. Teotia, S. Kadam, A. K. Singh, S. K. Verma, A. Bahulekar, S. Kanetkar and J. Bellare, Mater. Sci. Eng., C, 2017, 77, 857866.

27 B. Lee, Y. Baek, M. Lee, D. H. Jeong, H. H. Lee, J. Yoon and Y. H. Kim, Nat. Commun., 2015, 6, 7109.

28 R. Lou, W. Yu, Y. Song, Y. Ren, H. Zheng, X. Guo, Y. Lin, G. Pan, X. Wang and X. Ma, Carbohydr. Polym., 2017, 155, 456-465.

29 A. Modi, S. K. Verma and J. Bellare, J. Colloid Interface Sci., 2018, 514, 750-759.

30 R. Teotia, S. K. Verma, D. Kalita, A. K. Singh, G. Dahe and J. Bellare, J. Mater. Sci., 2017, 52, 12513-12523.

31 M. Amirilargani, A. Sabetghadam and T. Mohammadi, Polym. Adv. Technol., 2012, 23, 398-407.

32 C. H. Kim, M. S. Khil, H. Y. Kim, H. U. Lee and K. Y. Jahng, J. Biomed. Mater. Res., Part B, 2006, 78, 283-290.

33 U. Nekanti, L. Mohanty, P. Venugopal, S. Balasubramanian, S. Totey and M. Ta, Stem Cell Res., 2010, 5, 244-254.

34 A. Varki, Glycobiology, 1993, 3, 97-130.

35 M. Sarkar, J. Liao, E. A. Kabat, T. Tanabe and G. Ashwell, J. Biol. Chem., 1979, 254, 3170-3174.

36 P. Stahl, P. H. Schlesinger, E. Sigardson, J. S. Rodman and Y. Lee, Cell, 1980, 19, 207-215.

37 K. Y. Chen, R. H. Kramer and E. Canellakis, Biochim. Biophys. Acta, Biomembr., 1978, 507, 107-118.

38 G. Ashwell and A. G. Morell, Advances in Enzymology and Related Areas of Molecular Biology, 2006, vol. 41, pp. 99-128. 39 S. Zhang, T. Liu, L. Chen, M. Ren, B. Zhang, Z. Wang and Y. Wang, J. Mater. Sci.: Mater. Med., 2012, 23, 2001-2011.

40 B. Deorosan and E. A. Nauman, Stem Cells Int., 2011, 2011, DOI: $10.4061 / 2011 / 429187,12$ pages. 